\title{
A determinantal expression and a recursive relation of the Delannoy numbers
}

\author{
Feng Qi \\ Institute of Mathematics, \\ Henan Polytechnic University, \\ Jiaozuo 454010, Henan, China; \\ School of Mathematical Sciences, \\ Tiangong University, Tianjin 300387, China \\ email: qifeng618@gmail.com, \\ qifeng618@hotmail.com, qifeng618@qq.com
}

To Professor Sen-Lin Xu, my PhD supervisor, on his 81th birthday anniversary

\begin{abstract}
In the paper, by a general and fundamental, but non-extensively circulated, formula for derivatives of a ratio of two differentiable functions and by a recursive relation of the Hessenberg determinant, the author finds a new determinantal expression and a new recursive relation of the Delannoy numbers. Consequently, the author derives a recursive relation for computing central Delannoy numbers in terms of related Delannoy numbers.
\end{abstract}

\section{Motivations}

The Delannoy numbers, denoted by $D(p, q)$ for $p, q \geq 0$, form an array of positive integers which are related to lattice paths enumeration and other problems in combinatorics. For more information on their history and status in combinatorics, please refer to [1] and closely related references therein.

2010 Mathematics Subject Classification: Primary 11B83; Secondary 05A15, 05A10, 11B75, 11C20, 60G50

Key words and phrases: Delannoy number; central Delannoy number; determinantal expression; recursive relation 
In $[1$, Section 2] and [7], the explicit formulas

$$
D(p, q)=\sum_{i=0}^{p}\left(\begin{array}{l}
p \\
i
\end{array}\right)\left(\begin{array}{l}
q \\
i
\end{array}\right) 2^{i} \text { and } D(p, q)=\sum_{i=0}^{q}\left(\begin{array}{l}
q \\
i
\end{array}\right)\left(\begin{array}{c}
p+q-i \\
q
\end{array}\right)
$$

were given. It is well known [7] that the Delannoy numbers $D(p, q)$ satisfy a simple recurrence

$$
D(p, q)=D(p-1, q)+D(p-1, q-1)+D(p, q-1)
$$

and can be generated by

$$
\frac{1}{1-x-y-x y}=\sum_{p, q=0}^{\infty} D(p, q) x^{p} y^{q}
$$

When taking $\mathfrak{n}=\mathrm{p}=\mathrm{q}$, the numbers $\mathrm{D}(\mathfrak{n})=\mathrm{D}(\mathfrak{n}, \mathfrak{n})$ are known [7] as central Delannoy numbers which have the generating function

$$
\frac{1}{\sqrt{1-6 x+x^{2}}}=\sum_{n=0}^{\infty} \mathrm{D}(\mathrm{n}) \mathrm{x}^{\mathrm{k}}=1+3 x+13 x^{2}+63 x^{3}+\cdots .
$$

In [6, Theorems 1.1 and 1.3], considering the generating function (1), among other things, the authors expressed central Delannoy numbers $D(n)$ by an integral

$$
D(n)=\frac{1}{\pi} \int_{3-2 \sqrt{2}}^{3+2 \sqrt{2}} \frac{1}{\sqrt{(t-3+2 \sqrt{2})(3+2 \sqrt{2}-t)}} \frac{1}{t^{n+1}} d t
$$

and by a determinant

$$
D(n)=(-1)^{n}\left|\begin{array}{ccccccc}
a_{1} & 1 & 0 & \cdots & 0 & 0 & 0 \\
a_{2} & a_{1} & 1 & \cdots & 0 & 0 & 0 \\
a_{3} & a_{2} & a_{1} & \cdots & 0 & 0 & 0 \\
\vdots & \vdots & \vdots & \ddots & \vdots & \vdots & \vdots \\
a_{n-2} & a_{n-3} & a_{n-4} & \cdots & a_{1} & 1 & 0 \\
a_{n-1} & a_{n-2} & a_{n-3} & \cdots & a_{2} & a_{1} & 1 \\
a_{n} & a_{n-1} & a_{n-2} & \cdots & a_{3} & a_{2} & a_{1}
\end{array}\right|
$$

for $n \in \mathbb{N}$, where

$$
a_{n}=\frac{(-1)^{n+1}}{6^{n}} \sum_{\ell=1}^{n}(-1)^{\ell} 6^{2 \ell} \frac{(2 \ell-3) ! !}{(2 \ell) ! !}\left(\begin{array}{c}
\ell \\
n-\ell
\end{array}\right) .
$$


Making use of the integral expression 2, the authors derived in $[5,6]$ some new analytic properties, including some product inequalities and determinantal inequalities, of central Delannoy numbers $D(n)$.

In this paper, by a general and fundamental, but non-extensively circulated, formula for derivatives of a ratio of two differentiable functions in [2, p. 40] and by a recursive relation of the Hessenberg determinant in [3, p. 222, Theorem], we find a new determinantal expression and a new recursive relation of the Delannoy numbers $D(p, q)$. Consequently, we derive a recursive relation for computing central Delannoy numbers $D(n)$ in terms of related Delannoy numbers $\mathrm{D}(\mathrm{p}, \mathrm{q})$.

\section{A determinantal expression of the Delannoy num- bers}

In this section, by virtue of a general and fundamental, but non-extensively circulated, formula for derivatives of a ratio of two differentiable functions in $[2$, p. 40$]$, we find a new determinantal expression of the Delannoy numbers $\mathrm{D}(\mathrm{p}, \mathrm{q})$.

Theorem 1 For $\mathrm{p}, \mathrm{q} \geq 0$, the Delannoy numbers $\mathrm{D}(\mathrm{p}, \mathrm{q})$ can be determinantally expressed by

$$
D(p, q)=\frac{(-1)^{q}}{q !}\left|L_{(q+1) \times 1}(p) \quad M_{(q+1) \times q}(p)\right|_{(q+1) \times(q+1)},
$$

where

$$
\begin{aligned}
& \mathrm{L}_{(\mathrm{q}+1) \times 1}(\mathrm{p})=\left(\langle p\rangle_{0},\langle\mathrm{p}\rangle_{1}, \ldots,\langle\mathrm{p}\rangle_{\mathrm{q}}\right)^{\top}, \\
& M_{(q+1) \times q}(p)=\left((-1)^{i-j}\left(\begin{array}{c}
i-1 \\
j-1
\end{array}\right)\langle p+1\rangle_{i-j}\right)_{\substack{1 \leq i \leq q+1 \\
1 \leq j \leq q}} \text {, } \\
& \langle z\rangle_{n}= \begin{cases}z(z-1) \cdots(z-n+1), & n \geq 1 ; \\
1, & n=0\end{cases}
\end{aligned}
$$

is known as the $\mathrm{n}$-th falling factorial of the number $z \in \mathbb{C}$, and $\mathrm{T}$ denotes the transpose of a matrix. Consequently, central Delannoy numbers $\mathrm{D}(\mathrm{n})$ for $\mathrm{n} \geq 0$ can be determinantally expressed as

$$
D(n)=\frac{(-1)^{n}}{n !}\left|L_{(n+1) \times 1}(n) \quad M_{(n+1) \times n}(n)\right|_{(n+1) \times(n+1)} .
$$


Proof. We recall a general and fundamental, but non-extensively circulated, formula for derivatives of a ratio of two differentiable functions. Let $u(t)$ and $v(t) \neq 0$ be two $n$-th differentiable functions for $n \in \mathbb{N}$. Exercise 5) in [2, p. 40] reads that the $n$-th derivative of the ratio $\frac{u(t)}{v(t)}$ can be computed by

$$
\frac{d^{n}}{d x^{n}}\left[\frac{u(t)}{v(t)}\right]=(-1)^{n} \frac{\left|W_{(n+1) \times(n+1)}(t)\right|}{v^{n+1}(t)},
$$

where $U_{(n+1) \times 1}(t)$ is an $(n+1) \times 1$ matrix whose elements satisfy $\mathfrak{u}_{k, 1}(t)=$ $\mathrm{u}^{(\mathrm{k}-1)}(\mathrm{t})$ for $1 \leq \mathrm{k} \leq \mathrm{n}+1, \mathrm{~V}_{(\mathrm{n}+1) \times \mathrm{n}}(\mathrm{t})$ is an $(\mathrm{n}+1) \times \mathrm{n}$ matrix whose elements meet $v_{i, j}(t)=\left(\begin{array}{c}i-1 \\ j-1\end{array}\right) v^{(i-j)}(t)$ for $1 \leq i \leq n+1$ and $1 \leq j \leq n$, and $\left|W_{(n+1) \times(n+1)}(t)\right|$ is the determinant of the $(n+1) \times(n+1)$ matrix

$$
W_{(n+1) \times(n+1)}(t)=\left(u_{(n+1) \times 1}(t) \quad V_{(n+1) \times n}(t)\right)_{(n+1) \times(n+1)} .
$$

It is easy to see that

$$
\frac{\partial^{p}}{\partial x^{p}}\left(\frac{1}{1-x-y-x y}\right)=\frac{p !(1+y)^{p}}{[1-x-(1+x) y]^{p+1}} .
$$

Making use of the formula (5) gives

$$
\begin{gathered}
\frac{\partial^{p+q}}{\partial y^{q} \partial x^{p}}\left(\frac{1}{1-x-y-x y}\right)=p ! \frac{\partial^{q}}{\partial y^{q}} \frac{(1+y)^{p}}{[1-x-(1+x) y]^{p+1}} \\
\times p ! \frac{(-1)^{q}}{[1-x-(1+x) y]^{(p+1)(q+1)}} \\
\times \mid \begin{array}{cc}
(1+y)^{p} & {[1-x-(1+x) y]^{p+1}} \\
\langle p\rangle_{1}(1+y)^{p-1} & (-1)^{1}\langle p+1\rangle_{1}(1+x)^{1}[1-x-(1+x) y]^{p} \\
\langle p\rangle_{2}(1+y)^{p-2} & (-1)^{2}\langle p+1\rangle_{2}(1+x)^{2}[1-x-(1+x) y]^{p-1} \\
\langle p\rangle_{3}(1+y)^{p-3} & (-1)^{3}\langle p+1\rangle_{3}(1+x)^{3}[1-x-(1+x) y]^{p-2} \\
\vdots & \vdots \\
\langle p\rangle_{q-2}(1+y)^{p-q+2} & (-1)^{q-2}\langle p+1\rangle_{q-2}(1+x)^{q-2}[1-x-(1+x) y]^{p-q+3} \\
\langle p\rangle_{q-1}(1+y)^{p-q+1} & (-1)^{q-1}\langle p+1\rangle_{q-1}(1+x)^{q-1}[1-x-(1+x) y]^{p-q+2} \\
\langle p\rangle_{q}(1+y)^{p-q} & (-1)^{q}\langle p+1\rangle_{q}(1+x)^{q}[1-x-(1+x) y]^{p-q+1}
\end{array}
\end{gathered}
$$




$$
\begin{aligned}
& 0 \\
& \left(\begin{array}{l}
1 \\
1
\end{array}\right)[1-x-(1+x) y]^{p+1} \\
& \left(\begin{array}{l}
2 \\
1
\end{array}\right)(-1)^{1}\langle p+1\rangle_{1}(1+x)^{1}[1-x-(1+x) y]^{p} \\
& \left(\begin{array}{l}
3 \\
1
\end{array}\right)(-1)^{2}\langle p+1\rangle_{2}(1+x)^{2}[1-x-(1+x) y]^{p-1} \\
& \left(\begin{array}{c}
q-2 \\
1
\end{array}\right)(-1)^{q-3}\langle p+1\rangle_{q-3}(1+x)^{q-3}[1-x-(1+x) y]^{p-q+4} \\
& \left(\begin{array}{c}
q-1 \\
1
\end{array}\right)(-1)^{q-2}\langle p+1\rangle_{q-2}(1+x)^{q-2}[1-x-(1+x) y]^{p-q+3} \\
& \left(\begin{array}{l}
q \\
1
\end{array}\right)(-1)^{q-1}\langle p+1\rangle_{q-1}(1+x)^{q-1}[1-x-(1+x) y]^{p-q+2} \\
& 0 \\
& 0 \\
& \left(\begin{array}{l}
2 \\
2
\end{array}\right)[1-x-(1+x) y]^{p+1} \\
& \left(\begin{array}{l}
3 \\
2
\end{array}\right)(-1)^{1}\langle p+1\rangle_{1}(1+x)^{1}[1-x-(1+x) y]^{p}
\end{aligned}
$$

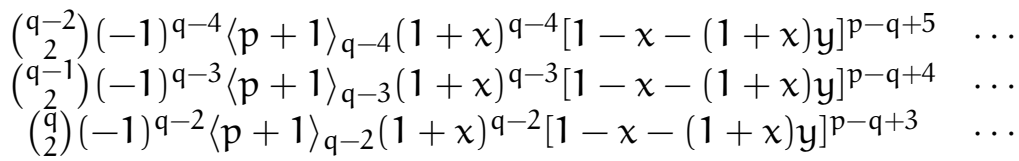

$$
\begin{aligned}
& 0 \\
& 0 \\
& 0 \\
& 0 \\
& \left(\begin{array}{l}
q-2 \\
q-2
\end{array}\right)[1-x-(1+x) y]^{p+1} \\
& \begin{array}{c}
\left(\begin{array}{c}
q-1 \\
q-2
\end{array}\right)(-1)\langle p+1\rangle_{1}(1+x)[1-x-(1+x) y]^{p} \\
\left(\begin{array}{c}
q \\
q-2
\end{array}\right)\langle p+1\rangle_{2}(1+x)^{2}[1-x-(1+x) y]^{p-1}
\end{array} \\
& 0 \\
& 0 \\
& 0 \\
& 0 \\
& \left(\begin{array}{l}
q-1 \\
q-1
\end{array}\right)[1-x-(1+x) y]^{p+1} \\
& \left(\begin{array}{c}
q \\
q-1
\end{array}\right)(-1)\langle p+1\rangle_{1}(1+x)[1-x-(1+x) y]^{p}
\end{aligned}
$$




$$
\begin{aligned}
& \rightarrow(-1)^{\mathrm{q}} p ! \mid \begin{array}{ccc}
\langle p\rangle_{0} & (-1)^{0}\langle p+1\rangle_{0} & 0 \\
\langle p\rangle_{1} & (-1)^{1}\langle p+1\rangle_{1} & \left(\begin{array}{l}
1 \\
1
\end{array}\right)(-1)^{0}\langle p+1\rangle_{0} \\
\langle p\rangle_{2} & (-1)^{2}\langle p+1\rangle_{2} & \left(\begin{array}{l}
2 \\
1
\end{array}\right)(-1)^{1}\langle p+1\rangle_{1} \\
\langle p\rangle_{3} & (-1)^{3}\langle p+1\rangle_{3} & \left(\begin{array}{l}
3 \\
1
\end{array}\right)(-1)^{2}\langle p+1\rangle_{2} \\
\vdots & \vdots & \vdots \\
\langle p\rangle_{q-2} & (-1)^{q-2}\langle p+1\rangle_{q-2} & \left(\begin{array}{c}
q \\
1
\end{array}\right)(-1)^{q-3}\langle p+1\rangle_{q-3} \\
\langle p\rangle_{q-1} & (-1)^{q-1}\langle p+1\rangle_{q}-1 & \left(\begin{array}{c}
q \\
q
\end{array}\right)(-1)^{q-2}\langle p+1\rangle_{q-2} \\
\langle p\rangle_{q} & (-1)^{q}\langle p+1\rangle_{q} & \left(\begin{array}{l}
q \\
1
\end{array}\right)(-1)^{q-1}\langle p+1\rangle_{q-1}
\end{array} \\
& \begin{array}{llll}
0 & \cdots & 0 & 0
\end{array} \\
& \begin{array}{llll}
0 & \cdots & 0 & 0
\end{array} \\
& \left(\begin{array}{llll}
2 \\
2
\end{array}\right)(-1)^{0}\langle p+1\rangle_{0} \quad \cdots \quad 00 \\
& \left(\begin{array}{l}
3 \\
2
\end{array}\right)(-1)^{1}\langle p+1\rangle_{1} \quad \cdots \quad 00 \\
& \begin{array}{clcc}
\left(\begin{array}{c}
q-2 \\
2
\end{array}\right)(-1)^{q-4}\langle p+1\rangle_{q-4} & \cdots & \left(\begin{array}{c}
q-2 \\
q-2
\end{array}\right)(-1)^{0}\langle p+1\rangle_{0} & 0 \\
\left(\begin{array}{c}
q-1 \\
2
\end{array}\right)(-1)^{q-3}\langle p+1\rangle_{q-3} & \cdots & \left(\begin{array}{c}
q-1 \\
q-2
\end{array}\right)(-1)^{1}\langle p+1\rangle_{1} & \left(\begin{array}{c}
q-1 \\
q-1
\end{array}\right)(-1)^{0}\langle p+1\rangle_{0} \\
\left(\begin{array}{c}
q \\
q
\end{array}\right)(-1)^{q-2}\langle p+1\rangle_{q-2} & \cdots & \left(\begin{array}{c}
q \\
q-2
\end{array}\right)(-1)^{2}\langle p+1\rangle_{2} & \left(\begin{array}{c}
q \\
q-1
\end{array}\right)(-1)^{1}\langle p+1\rangle_{1}
\end{array}
\end{aligned}
$$

as $x, y \rightarrow 0$. Consequently, we have

$$
\begin{aligned}
D(p, q) & =\frac{1}{p ! q !} \frac{\partial^{p+q}}{\partial y^{q} \partial x^{p}}\left(\frac{1}{1-x-y-x y}\right) \\
= & \frac{(-1)^{q}}{q !}\left|\left(\langle p\rangle_{i j}\right)_{\substack{0 \leq i \leq q \\
j=1}}\left((-1)^{i-j\left(\begin{array}{c}
i-1 \\
j-1
\end{array}\right)\langle p+1\rangle_{i-j}}\right)_{\substack{1 \leq i \leq q+1 \\
1 \leq j \leq q}}\right|_{(q+1) \times(q+1)} .
\end{aligned}
$$

The determinantal expression (3) is thus proved.

From (3), we readily see that, when $\mathfrak{n}=\mathrm{p}=\mathrm{q}$, central Delannoy numbers $D(n)$ for $n \geq 0$ can be expressed as (4). The proof of Theorem 1 is complete.

\section{A recursive relation of the Delannoy numbers}

In this section, by virtue of a recursive relation of the Hessenberg determinant in $[3$, p. 222 , Theorem], we find a recursive relation of the Delannoy numbers $\mathrm{D}(\mathrm{p}, \mathrm{q})$.

Theorem 2 For $\mathrm{p}, \mathrm{q} \geq 0$, the Delannoy numbers $\mathrm{D}(\mathrm{p}, \mathrm{q})$ satisfy the recursive relation

$$
D(p, q)=\left(\begin{array}{l}
p \\
q
\end{array}\right)+(-1)^{q-1} \sum_{r=0}^{q-1}(-1)^{r}\left(\begin{array}{l}
p+1 \\
q-r
\end{array}\right) D(p, r) .
$$


Consequently, central Delannoy numbers $\mathrm{D}(\mathrm{n})$ for $\mathrm{n} \geq 0$ satisfy

$$
D(n)=1+(-1)^{n+1} \sum_{r=0}^{n-1}(-1)^{r}\left(\begin{array}{c}
n+1 \\
r+1
\end{array}\right) D(n, r) .
$$

Proof. Let $\mathrm{Q}_{0}=1$ and

$$
Q_{n}=\left|\begin{array}{cccccc}
e_{1,1} & e_{1,2} & 0 & \ldots & 0 & 0 \\
e_{2,1} & e_{2,2} & e_{2,3} & \ldots & 0 & 0 \\
e_{3,1} & e_{3,2} & e_{3,3} & \ldots & 0 & 0 \\
\vdots & \vdots & \vdots & \vdots & \vdots & \vdots \\
e_{n-2,1} & e_{n-2,2} & e_{n-2,3} & \ldots & e_{n-2, n-1} & 0 \\
e_{n-1,1} & e_{n-1,2} & e_{n-1,3} & \ldots & e_{n-1, n-1} & e_{n-1, n} \\
e_{n, 1} & e_{n, 2} & e_{n, 3} & \ldots & e_{n, n-1} & e_{n, n}
\end{array}\right|
$$

for $n \in \mathbb{N}$. In [3, p. 222, Theorem], it was proved that the sequence $Q_{n}$ for $\mathrm{n} \geq 0$ satisfies $\mathrm{Q}_{1}=\mathrm{e}_{1,1}$ and

$$
Q_{n}=\sum_{r=1}^{n}(-1)^{n-r} e_{n, r}\left(\prod_{j=r}^{n-1} e_{j, j+1}\right) Q_{r-1}
$$

for $n \geq 2$, where the empty product is understood to be 1 . Replacing the determinant $Q_{r}$ by $(-1)^{r-1}(r-1) ! D(p, r-1)$ in $(3)$ for $1 \leq r \leq n$ in the recursive relation (8) and simplifying give

$$
D(p, n-1)=\frac{\langle p\rangle_{n-1}}{(n-1) !}+(-1)^{n} \sum_{r=2}^{n}(-1)^{r} \frac{\langle p+1\rangle_{n-r+1}}{(n-r+1) !} D(p, r-2)
$$

which is equivalent to the recursive relation (6).

When $\mathrm{n}=\mathrm{p}=\mathrm{q}$ in (6), we can see that central Delannoy numbers $\mathrm{D}(\mathrm{n})$ satisfy the recursive relation (7). The proof of Theorem 2 is complete.

Remark 1 This paper is a shortened version of the electronic preprint [4].

\section{Acknowledgements}

The author thanks anonymous referees for their careful corrections and valuable comments on the original version of this paper. 


\section{References}

[1] C. Banderier and S. Schwer, Why Delannoy numbers? J. Statist. Plann. Inference, 135 (2005), no. 1, 40-54; available online at https://doi.org/10.1016/j.jspi.2005.02.004.

[2] N. Bourbaki, Elements of Mathematics: Functions of a Real Variable: Elementary Theory, Translated from the 1976 French original by Philip Spain. Elements of Mathematics (Berlin). Springer-Verlag, Berlin, 2004; available online at http://dx.doi.org/10.1007/978-3-642-59315-4.

[3] N. D. Cahill and D. A. Narayan, Fibonacci and Lucas numbers as tridiagonal matrix determinants, Fibonacci Quart., 42 (2004), no. 3, 216-221.

[4] F. Qi, A determinantal expression and a recursive relation of the Delannoy numbers, arXiv preprint (2020), available online at https://arxiv.org/abs/2003.12572.

[5] F. Qi, V. Čerňanová, and Y. S. Semenov, Some tridiagonal determinants related to central Delannoy numbers, the Chebyshev polynomials, and the Fibonacci polynomials, Politehn. Univ. Bucharest Sci. Bull. Ser. A Appl. Math. Phys., 81 (2019), no. 1, 123-136.

[6] F. Qi, V. Čerňanová, X.-T. Shi, and B.-N. Guo, Some properties of central Delannoy numbers, J. Comput. Appl. Math., 328 (2018), 101-115; available online at https://doi.org/10.1016/j.cam.2017.07.013.

[7] Y. Wang, S.-N. Zheng, and X. Chen, Analytic aspects of Delannoy numbers, Discrete Math., 342 (2019), no. 8, 2270-2277; available online at https://doi.org/10.1016/j.disc.2019.04.003. 